\title{
Oxidative Phosphorylation Is Dysregulated Within the Basocortical Circuit in a 6-month old Mouse Model of Down Syndrome and Alzheimer's Disease
}

\author{
Melissa J. Alldred ${ }^{1,2}$, Sang Han Lee ${ }^{3,4}$, Grace E. Stutzmann ${ }^{5}$ \\ and Stephen $D$. Ginsberg $1,2,6,7$ *
}

'Center for Dementia Research, Nathan Kline Institute, Orangeburg, NY, United States, ${ }^{2}$ Departments of Psychiatry, New York University Grossman School of Medicine, New York, NY, United States, ${ }^{3} \mathrm{Center}$ for Biomedical Imaging and Neuromodulation, Nathan Kline Institute, Orangeburg, NY, United States, ${ }^{4}$ Department of Child and Adolescent Psychiatry, New York University Grossman School of Medicine, New York, NY, United States, ${ }^{5}$ Center for Neurodegenerative Disease and Therapeutics, Discipline of Neuroscience, Rosalind Franklin University/The Chicago Medical School, North Chicago, IL, United States, ${ }^{6}$ Neuroscience \& Physiology, New York University Grossman School of Medicine, New York, NY, United States, ${ }^{7}$ NYU Neuroscience Institute, New York University Grossman School of Medicine, New York, NY, United States

\section{OPEN ACCESS}

Edited by:

Christian J. Pike, University of Southern California, United States

Reviewed by: Fabio Di Domenico, Sapienza University of Rome, Italy Marie-Claude Potier, Centre National de la Recherche Scientifique (CNRS), France

*Correspondence: Stephen D. Ginsberg ginsberg@nki.rfmh.org

Received: 11 May 2021 Accepted: 13 July 2021 Published: 19 August 2021

Citation: Alldred MJ, Lee SH, Stutzmann GE and Ginsberg SD (2021) Oxidative

Phosphorylation Is Dysregulated Within the Basocortical Circuit in a 6-month old Mouse Model of Down Syndrome and Alzheimer's Disease. Front. Aging Neurosci. 13:707950. doi: 10.3389/fnagi.2021.707950
Down syndrome (DS) is the primary genetic cause of intellectual disability (ID), which is due to the triplication of human chromosome 21 (HSA21). In addition to ID, HSA21 trisomy results in a number of neurological and physiological pathologies in individuals with DS, including progressive cognitive dysfunction and learning and memory deficits which worsen with age. Further exacerbating neurological dysfunction associated with DS is the concomitant basal forebrain cholinergic neuron (BFCN) degeneration and onset of Alzheimer's disease (AD) pathology in early mid-life. Recent single population RNA sequencing (RNA-seq) analysis in the Ts65Dn mouse model of DS, specifically the medial septal cholinergic neurons of the basal forebrain (BF), revealed the mitochondrial oxidative phosphorylation pathway was significantly impacted, with a large subset of genes within this pathway being downregulated. We further queried oxidative phosphorylation pathway dysregulation in Ts65Dn mice by examining genes and encoded proteins within brain regions comprising the basocortical system at the start of BFCN degeneration (6 months of age). In select Ts65Dn mice we demonstrate significant deficits in gene and/or encoded protein levels of Complex I-V of the mitochondrial oxidative phosphorylation pathway in the BF. In the frontal cortex ( $F r \mathrm{Ctx}$ ) these complexes had concomitant alterations in select gene expression but not of the proteins queried from Complex I-V, suggesting that defects at this time point in the BF are more severe and occur prior to cortical dysfunction within the basocortical circuit. We propose dysregulation within mitochondrial oxidative phosphorylation complexes is an early marker of cognitive decline onset and specifically linked to BFCN degeneration that may propagate pathology throughout cortical memory and executive function circuits in $D S$ and $A D$.

Keywords: oxidative phosphorylation, basal forebrain, Down syndrome, Alzheimer's disease, selective vulnerability 


\section{INTRODUCTION}

Down syndrome (DS) is caused by the triplication of human chromosome 21 (HSA21) and is the primary genetic cause of intellectual disability (ID). HSA21 triplication is present in approximately 1 in 700 live births and these individuals exhibit multiple systemic functional deficits, including heart conditions, increased incidence of leukemias, epilepsy, premature aging, and neurological deficits (Bittles et al., 2007; So et al., 2007; Lott, 2012; Presson et al., 2013; Mai et al., 2019). While the lifespan of individuals with DS has increased significantly in the past several decades, healthspan lags appreciably behind (Hill et al., 2003; Bittles et al., 2007; Presson et al., 2013; Dick et al., 2016). Most adults with DS start to exhibit Alzheimer's disease (AD)-like pathology, including senile plaques, neurofibrillary tangles, synaptic dysfunction, and basal forebrain cholinergic neuron (BFCN) degeneration, with the accompanying cognitive decline, typically by the mid-third decade of life (Mann et al., 1984; Coyle et al., 1988; Beacher et al., 2009; Lott and Dierssen, 2010; Costa, 2012; Lott, 2012; Hartley et al., 2015; Annus et al., 2016). BFCN degeneration is an early pathological feature of both $\mathrm{DS}$ and $\mathrm{AD}$ and coincides with cognitive decline early in disease onset and throughout progression (Yates et al., 1980; Sendera et al., 2000; Mufson et al., 2002, 2008; Iulita et al., 2014). Recent imaging evidence indicates volume reductions in the basal forebrain $(\mathrm{BF})$ predicts entorhinal cortex loss and cortical spread of degeneration in $\mathrm{AD}$ and correlates with $\mathrm{AD}$ biomarkers (Grothe et al., 2013; Cavedo et al., 2020; Fernández-Cabello et al., 2020; Teipel et al., 2020), suggesting BF dysfunction is one of the earliest pathological changes during the development of $\mathrm{AD}$.

Mitochondrial oxidative phosphorylation is the main energy source within neurons and is critical for normal brain development and function (Mattson et al., 2008; Hall et al., 2012). Deficits within the oxidative phosphorylation pathway have devastating effects on normal neuronal function (Mattson et al., 2008). The oxidative phosphorylation apparatus has five mitochondrial respiratory chain complexes (Complexes I-V), comprised of NADH-ubiquinone oxidoreductase Complex I, succinate dehydrogenase Complex II, cytochrome $\mathrm{bc}_{\mathrm{L}}$ Complex III, cytochrome C oxidase Complex IV, and the ATP synthase Complex V (Valenti et al., 2014). Individual mitochondrial complex proteins within the oxidative phosphorylation pathway have been shown to be dysregulated in DS cell culture models (Valenti et al., 2016; Briggs et al., 2017). Little information is currently available on the status of individual oxidative phosphorylation markers in the context of DS in vivo (Kim et al., 2000, 2001; Bambrick and Fiskum, 2008). Although little doubt exists that oxidative phosphorylation is critical for normal brain function, oxidative phosphorylation pathway deficits in the DS brain demands further exploration. Indeed, recent evidence suggests evaluating oxidative phosphorylation complex proteins in the cortex of the well-established Ts65Dn mouse model of DS and AD is difficult and variable (Lanzillotta et al., 2021), further highlighting the necessity of additional interrogation of the oxidative phosphorylation pathway in the brain of DS and AD relevant model systems.
There are several mouse models of DS available, however, the Ts65Dn mouse is the most prevalent in terms of use and applicability (Rueda et al., 2012; Ruparelia et al., 2012; Ahmed et al., 2017). The Ts65Dn mouse model recapitulates many of the endophenotypes of $\mathrm{DS}$ and $\mathrm{AD}$, including hippocampal-dependent learning and memory deficits, BFCN degeneration and septohippocampal circuit dysfunction, notably CA1 pyramidal neuron and choline acetyltransferase (ChAT) activity deficits (Granholm et al., 2000; Belichenko et al., 2004, 2009; Kelley et al., 2014a,b). Degeneration of the BFCN system, including within the septohippocampal and basocortical pathways, are hallmarks of disease progression in $\mathrm{DS}$ and $\mathrm{AD}$ and are a primary feature of the Ts65Dn mouse model (Holtzman et al., 1996; Granholm et al., 2000; Hunter et al., 2003a; Strupp et al., 2016). BFCN degeneration begins at approximately 6 months of age (MO) in Ts65Dn mice, and loss of BFCNs and deficits in hippocampal cholinergic innervation are uniformly reported by $\sim 10$ MO (Holtzman et al., 1996; Cooper et al., 2001; Hunter et al., 2003b; Contestabile et al., 2006; Powers et al., 2016). Many of the mitochondrial deficits reported in human DS, principally in the periphery (Izzo et al., 2018; BayonaBafaluy et al., 2021) are also present in this trisomic model including lower ATP production in vitro (Valenti et al., 2016) and metabolic changes in peripheral cell types (Cisterna et al., 2020). Further, genes postulated to be involved in mitochondrial dysfunction, including Dyrk1a and Ets2 (Izzo et al., 2018) are triplicated in human DS and reproduced in the Ts65Dn mouse model. These two genes have recently been shown to be upregulated within BFCNs microisolated from the medial septal nucleus (MSN; Alldred et al., 2021).

Although the oxidative phosphorylation pathway is known to be dysregulated in DS (Helguera et al., 2013; Izzo et al., 2018; Bayona-Bafaluy et al., 2021) and changes in oxidative phosphorylation states have significant deleterious effects on normal neuronal function (Mattson et al., 2008), prior studies lack pathway-based evaluations conducted in vivo to understand mechanisms driving this critical pathology. Herein, we utilized the Ts65Dn mouse model to examine connectivity based degeneration deficits in two interconnected brain regions comprising the basocortical system, the BF and Fr Ctx, which are critically impacted in $\mathrm{DS}$ and $\mathrm{AD}$ and are reflective of age-related cognitive decline and loss of executive function. We examined $\mathrm{BF}$ and Fr Ctx levels for oxidative phosphorylation changes at the transcript and encoded protein levels within each oxidative phosphorylation complex. We postulate reductions observed within the basocortical circuit indicate $\mathrm{BF}$ degeneration drives oxidative phosphorylation dysregulation and paces or precedes cortical dysfunction associated with DS and AD.

\section{MATERIALS AND METHODS}

\section{Mice}

Animal protocols were approved by the Nathan Kline Institute/NYU Grossman School of Medicine IACUC in accordance with NIH guidelines. Breeder pairs (female Ts65Dn and male C57Bl/6J Eicher x C3H/HeSnJ F1 mice) were purchased 
from Jackson Laboratories (Bar Harbor, ME, USA) and mated at the Nathan Kline Institute. Mice were given ad libitum food and water access (Alldred et al., 2015a,b). Standard cages contained paper bedding and several objects for enrichment (e.g., plastic igloo, t-tube, and cotton square). Mice were maintained on a 12-h light-dark cycle under temperature- and humiditycontrolled conditions. Tail clips were taken and pups were genotyped (Duchon et al., 2011) at weaning (P21) and aged to $\sim 6 \mathrm{MO}$.

\section{Tissue Preparation}

At $\sim 6 \mathrm{MO}$, mice were sacrificed for brain tissue accession. Mice were given an overdose of ketamine and xylazine and perfused transcardially with ice-cold $0.15 \mathrm{M}$ phosphate buffer (Alldred et al., 2015a,b, 2018, 2019). Brain tissues were accessed from Ts65Dn (Ts; $n=10$ ) and age-matched normal disomic $(2 \mathrm{~N} ; n=10)$ male mice, with littermates between $2 \mathrm{~N}$ and $\mathrm{Ts}$ mice used when possible (age range: 5.8-6.4 MO, mean age 6.0 MO). The BF was dissected to enrich for cholinergic neurons in the medial septal/ventral diagonal band region $(\sim$ Bregma 1.35-0.26) as well as the left Fr Ctx dissected using standard coordinates from the mouse brain atlas (Paxinos and Franklin, 2001). Dissections were either flash-frozen or kept on wet ice for homogenization directly following brain accrual. Tissue was homogenized using ice-cold Tris homogenization buffer [THB; $20 \mathrm{mM}$ Tris-Cl (pH 7.4), $1 \mathrm{mM}$ EGTA, $1 \mathrm{mM}$ EDTA and $0.25 \mathrm{M}$ sucrose] with a protease inhibitor cocktail (1:1,000, I3786, SigmaAldrich, St. Louis, MO, USA and $1 \mathrm{mM}$ PMSF, ThermoFisher, Waltham, MA, USA) using $1.5 \mathrm{~mm}$ zirconium beads on Beadbug homogenizer (Benchmark Scientific, Sayreville, NJ, USA) for $30 \mathrm{~s}$ at 4,000 rpm. Post homogenization, samples were kept on ice and cell debris was spun down at 2,500× $\mathrm{g}$ for $5 \mathrm{~min}$ at $4^{\circ} \mathrm{C}$. The supernatant was aliquoted to fresh tubes for isolation of RNA (done immediately following homogenization) or protein assays (each assay done with fresh aliquots of tissue homogenates stored at $-80^{\circ} \mathrm{C}$ ). RNase-free precautions were employed, and solutions were made with 18.2 mega Ohm RNase-free water (Nanopure Diamond, Barnstead, Dubuque, IA, USA).

\section{RNA Purification}

RNA from microdissected regions of mouse tissue from BF and Fr Ctx were purified using the miRNeasy Mini kit (Qiagen, Hilden, Germany) according to manufacturers' specifications. A DNase digestion was performed twice sequentially before the final washes and RNA purification (Alldred et al., 2021). RNA quality control was performed at a 1:5 dilution to preserve RNA for downstream applications (RNA 6000 pico kit, Agilent, Santa Clara, CA, USA).

\section{RT-qPCR}

Equal amounts of RNA was reverse transcribed in a $50 \mu \mathrm{l}$ reaction volume to generate cDNA from Fr Ctx and BF tissue from $\mathrm{Ts}$ and $2 \mathrm{~N}$ littermates $(n=10$ per genotype per brain region) using random hexamers as described previously (Alldred et al., 2012, 2015a,b, 2018, 2019; Bordi et al., 2016). RT-qPCR was performed using $1 \mu \mathrm{l}$ of $\mathrm{cDNA}$ and Taqman PCR primers for select genes from oxidative phosphorylation Complexes I-V along with a mitochondrial rRNA gene (Table 1) to assay samples in triplicate on a real-time qPCR cycler (PikoReal, ThermoFisher) as previously described (Alldred et al., 2008, 2012, 2015a,b, 2018, 2019; Jiang et al., 2010). The ddCT method was used to determine relative gene level differences between genotypes (ABI, 2004; Ginsberg et al., 2010; Jiang et al., 2010). Glucuronidase beta (Gusb, Mm01197678_m1) and $45 \mathrm{~S}$ pre-ribosomal RNA (Rn45s; Table 1) qPCR products were interrogated for use as controls as they did not show significant changes by genotype within MSN BFCN RNA-seq data (Alldred et al., 2021). Rn45s was subsequently selected as the control housekeeping gene. Negative controls consisted of the reaction mixture without input RNA. For each gene, the PCR product synthesis was modeled as a function of genotype, using mixed effects models with random mouse effect to account for the correlation between repeated assays on the same mouse (McCulloch et al., 2011; Alldred et al., 2015a,b, $2018,2019)$. Significance was judged at the level $\alpha=0.05$, twosided.

\section{Protein Assays}

Protein expression analysis was performed using the WES system (Protein Simple, Santa Clara, CA, USA; Nguyen et al., 2011). Briefly, protein samples were diluted in THB buffer 1:100 (w/v), with $1 \times$ final concentration of fluorescent molecular weight marker (provided in the kit) and heated to $50^{\circ} \mathrm{C}$ for $5 \mathrm{~min}$ (as per manufacturers' recommendation), then cooled to $4^{\circ} \mathrm{C}$ before loading onto the WES system plate with a molecular weight ladder. All blocking reagents, chemiluminescent substrate, separation, and stacking matrices (Protein Simple) were dispensed to designated wells. Primary antibodies against Complex I-V (Total OXPHOS panel, 1:20 dilution, ab110413, AbCam, Cambridge, United Kingdom) containing five mouse mAbs, Complex I (NADH:ubiquinone oxidoreductase subunit B8; NDUFB8), Complex II (succinate dehydrogenase beta; SDHB), Complex III (Cytochrome b-c1 complex subunit 2;UQCR2), Complex IV (Mitochondrially encoded cytochrome C oxidase I; MTCO1) and Complex V (ATP synthase lipidbinding protein; ATP5A), along with a control antibody against $\beta$-Tubulin III ( $\beta$-TubIII; R\&D Systems, Minneapolis, $M N$, USA, MAB1195 1:50) and HRP conjugated secondary antibody (rabbit anti-mouse; DM-002; Protein Simple) were dispensed to designated wells. Plates were spun for $5 \mathrm{~min}$ at $1,000 \times g$ and loaded onto the WES unit, where separation electrophoresis and immunodetection steps are fully automated within the capillary system. Instrument default settings were used with the exception of protein loading run time that was increased from 25 to 35 min. The digital image was analyzed with Compass software (Protein Simple), utilizing dropped lines for peak analysis area calculation. Detected proteins were compared to control protein ( $\beta$-TubIII) and reported as the normalized percentage of control. Each protein was performed in triplicate on separate plate runs. Statistical analysis was conducted on each protein normalized to $\beta$-TubIII and modeled as a function of the mouse study group (Ts and $2 \mathrm{~N}, n=10$ per genotype per brain region), using mixed effects models with random mouse effect to account for the correlation between repeated assays on the same mouse 
TABLE 1 | List of TaqMan primers for RT-qPCR analysis.

\begin{tabular}{|c|c|c|}
\hline Gene & TaqMan Primer & Description \\
\hline Rn45S & Rn03928990_g1 & Housekeeping, 45S pre-ribosomal RNA \\
\hline Mt-Nd1 & Mm04225274_s1 & Complex I, mitochondrial NADH dehydrogenase 1 \\
\hline Mt-Nd4I & Mm04225294_s1 & Complex I, mitochondrial NADH $4 \mathrm{~L}$ dehydrogenase \\
\hline Sdha & Mm01352360_m1 & Complex II, succinate dehydrogenase complex flavoprotein, subunit A \\
\hline Mt-Cyt $\beta$ & Mm04225271_g1 & Complex III, mitochondrial cytochrome b \\
\hline Mt-Cox2 & Mm03294838_g1 & Complex IV, mitochondrial Cytochrome c oxidase subunit II \\
\hline Mt-Atp8 & Mm04225236_g1 & Complex V, mitochondrial ATP synthase 8 \\
\hline Mt-Rnr1 & Mm04260177_s1 & Mitochondrial 12S rRNA \\
\hline
\end{tabular}

(McCulloch et al., 2011; Alldred et al., 2015a,b, 2018, 2019). Significance was judged at the level $\alpha=0.05$, two-sided.

\section{Deproteinization and ATP Assay}

Biochemical analysis of ATP levels were conducted using Fr Ctx tissue. Deproteinization of mouse Fr Ctx homogenates were performed utilizing the Deproteinizing Sample preparation kit (ab204708, AbCam) according to manufacturer's specifications with the following alterations, starting sample volume was reduced to $50 \mu \mathrm{l}$ from $100 \mu \mathrm{l}$ with $7.5 \mu \mathrm{l}$ of TCA, and neutralization was performed with $5 \mu \mathrm{l}$ of neutralization solution. Immediately following deproteinization, an ATP assay (ATP assay kit, ab83355, AbCam) was performed in duplicate for each sample ( $n=6$ per genotype) utilizing a 1:4 dilution of the deproteinized sample according to the manufacturer's specifications for the fluorometric assay. The fluorometric samples were read in duplicate on a plate reader (SpectraMax, Molecular Devices, San Jose, CA, USA). ATP concentration was calculated according to the manufacturer's specifications. Statistical analysis was performed using a non-parametric method (Wilcoxon Test) due to the small sample size and some losses of the sample (Wilcoxon, 1946; Pratt, 1959). Significance was judged at the level $\alpha=0.05$, two-sided.

\section{RESULTS}

To understand changes in oxidative phosphorylation within the basocortical circuit, we examined RNA and protein levels from each of the five complexes in the oxidative phosphorylation pathway from two synaptically connected brain regions critical for attention, memory, and executive function that show a significant decline in $\mathrm{DS}$ and $\mathrm{AD}$. This examination was based upon gene expression profile changes in the oxidative phosphorylation pathway from MSN BFCNs by single population RNA-seq in $\sim 6 \mathrm{MO}$ Ts65Dn mice compared to $2 \mathrm{~N}$ littermates (Alldred et al., 2021). Bioinformatic inquiry by Kyoto Encyclopedia of Genes and Genomes (KEGG) analysis of the oxidative phosphorylation pathway revealed downregulation of multiple subunits of Complex I, III, IV, and V, but no significant changes in Complex II subunits within MSN BFCNs (Figure 1).

To examine if transcriptomic changes seen in the Ts65Dn MSN BFCNs are selective to the MSN enriched BF or are seen throughout the basocortical circuit, we examined both $\mathrm{BF}$ and Fr Ctx at the RNA and protein level for expression changes at the start of BFCN degeneration. We examined a total of six subunits from Complexes I-V within the oxidative phosphorylation pathway for gene expression, utilizing transcripts that were significantly downregulated in MSN BFCNs via single population RNA-seq along with candidates that were not differentially regulated, to examine the pathway in a comprehensive manner. We also assessed the Mt-Rnrl gene, which encodes the $12 \mathrm{~S}$ rRNA as a marker of total mitochondrial RNA. In the BF, downregulation was observed for members of Complex I, Mt-Nd1 (trend level $p=0.072$ ) and $M t-N d 4 l$ $(p<0.00228)$, Complex II, Sdha $(p<0.00846)$, Complex III, $M t$ Cyt $\beta$ (not significant), Complex IV, $M t$-Cox2 (not significant), and Complex V, Mt-Atp8 $(p<0.0268$; Figure 2A). Total mitochondrial rRNA (Mt-Rnr1) also showed a downregulated trend $(p=0.064$; Figure $2 \mathrm{~A})$. When correlating the single population MSN BFCN RNA-seq to regional BF RT-qPCR log fold changes (LFC), a moderately high correlation was observed $\left(R^{2}=0.5759\right.$; Figure 2B $)$. Interestingly, while not significant, downregulation observed via RT-qPCR for $M t-N d 1$ and $M t$ Cox2 correlated with the significant downregulation seen in trisomic MSN BFCNs by RNA-seq. The regional BF gene expression of $M t-N d 1$ was only trend level downregulated via RT-qPCR, suggesting these changes are neuron-specific and the observed downregulation within MSN BFCNs is diluted when examined in tissue with admixed neuronal and non-neuronal cell types. The same subset of genes was interrogated in the Fr Ctx of Ts65Dn mice compared to $2 \mathrm{~N}$ littermates by RTqPCR. Of the six genes examined from the five complexes, no changes were found in either Complex I or II genes ( $M t$ $N d 1, M t-N d 4 l$, and Sdha), trend level decreases in Complex III and IV genes (Mt-Cyt $\beta p=0.067$ and Mt-Cox2 $p=0.079)$, and significant downregulation of Complex $\mathrm{V}$ member $M t$ Atp8 $(p<0.0059$; Figure 2C). Total mitochondrial rRNA was significantly downregulated in the trisomic Fr Ctx $(p<0.0012$; Figure 2C). Similar to regional BF RT-qPCR, a moderately high correlation between MSN BFCN RNA-seq and Fr Ctx RT-qPCR LFC was found $\left(R^{2}=0.6679\right.$; Figure 2D).

We employed the Total OXPHOS panel (Abcam) to assess protein expression of select subunits from Complexes $\mathrm{I}-\mathrm{V}$ in the $\mathrm{BF}$ and $\mathrm{Fr}$ Ctx within Ts65Dn mice and $2 \mathrm{~N}$ littermates. When examining the digital traces in $\mathrm{BF}$ homogenates (Figure 3A), we saw a relatively high expression of Complex II-V proteins, but relatively low expression of Complex I protein (NDUFB8), indicating this may not be a major subunit expressed in basocortical brain tissue. Within 


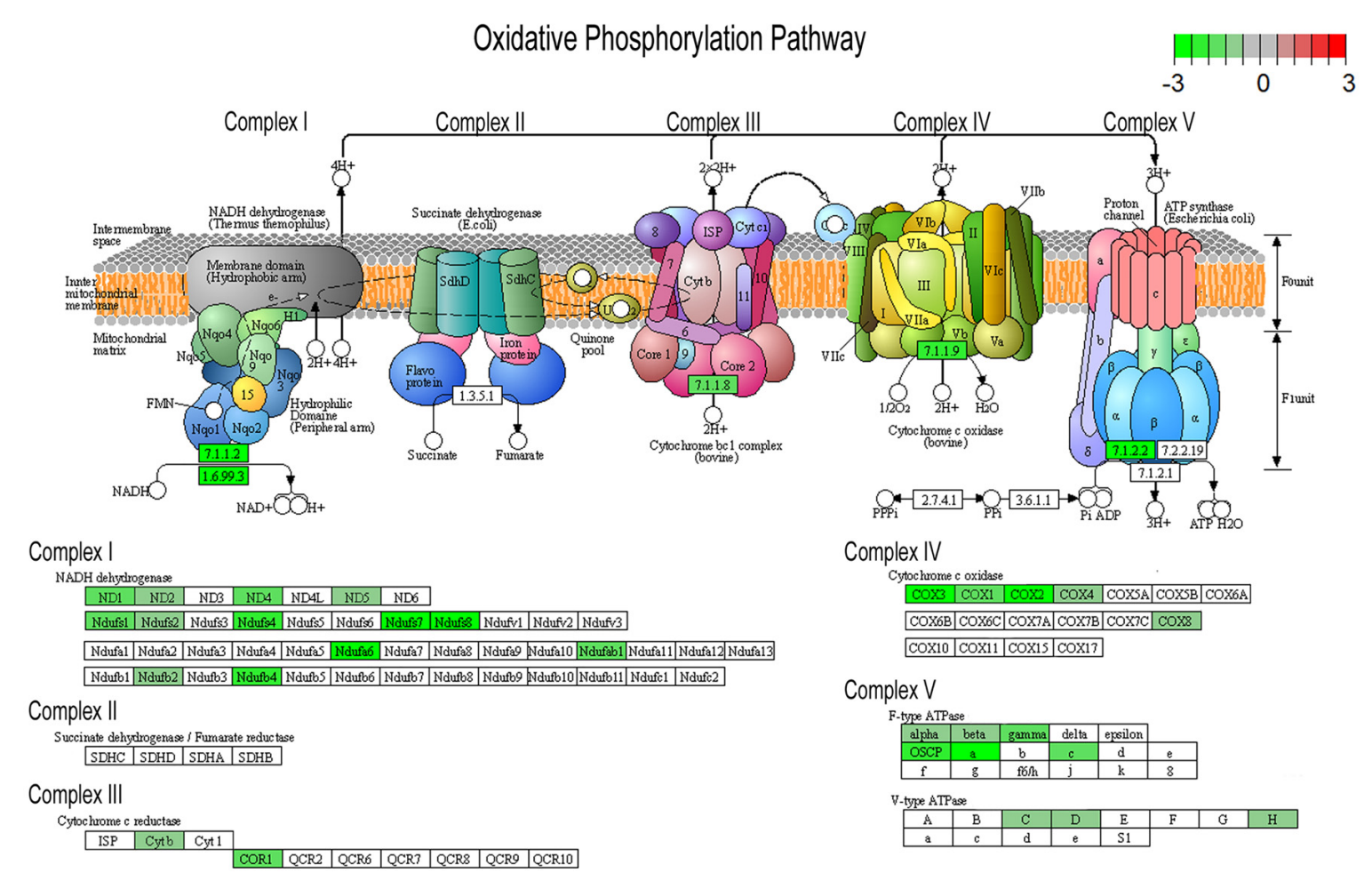

Data on KEGG graph

FIGURE 1 | Pronounced downregulation of members of the oxidative phosphorylation pathway in trisomic basal forebrain cholinergic neurons (BFCNs). The Kyoto Encyclopedia of Genes and Genomes (KEGG) derived oxidative phosphorylation pathway is rendered by Pathview, with significantly dysregulated genes highlighted for each complex from the medial septal nucleus (MSN) BFCNs as assayed by single population RNA-seq (Alldred et al., 2021). Downregulation is shown as color coded log fold change difference (LFC) with green depicting downregulation and red depicting upregulation. Note no genes within the oxidative phosphorylation pathway were upregulated.

the BF, Complex I, NDUFB8 expression was not significantly downregulated. However, downregulation of proteins from Complex II (SDHB, $p<0.016$ ), III (UQCRC2, $p<0.0055$ ), IV (MT-CO1, $p<0.0034$ ), and V (ATP5A, $p<0.019$ ) were found (Figure 3B), which highly correlated with MSN BFCN RNA-seq data $\left(R^{2}=0.7658\right.$; Figure $\left.3 C\right)$. When examining the same Complex I-V proteins in Fr Ctx tissue, variability in relative expression levels was observed between animals (Figure 3D) and no significant downregulation of protein expression was detected. The only significant change in protein expression in trisomic Fr Ctx tissue homogenates was upregulation of Complex I (NDUFB8, $p<0.0012$; Figure 3E). In contrast to RT-qPCR findings, protein levels in Fr Ctx did not correlate with MSN BFCN RNA-seq data $\left(R^{2}=0.0422\right.$, Figure 3F).

We indirectly examined the functionality of the oxidative phosphorylation complex by biochemical analysis of ATP levels, which are the output of the ATP synthase Complex V. Due to the small size of mouse BF and the quantity of tissue needed for the ATP assay, we were only able to perform this measurement in Fr Ctx tissue. We found a significant decrease in ATP levels in trisomic Fr Ctx ( $p<0.0307$; Figure 3G), which matched Fr Ctx RT-qPCR findings for Complex V.

\section{DISCUSSION}

We employed the Ts65Dn mouse model of DS and AD to examine oxidative phosphorylation pathway changes within the basocortical circuit. The BF provides the main cholinergic inputs for the hippocampus and cerebral cortex (Mesulam et al., 1983). Cholinergic fiber input into the cortex is involved in both attentional behavior and cognition and the activity of these cholinergic neurons is decreased during normal human aging (Mufson et al., 2003). This deficit is exacerbated during $\mathrm{AD}$ and DS progression (Coyle et al., 1986, 1988; Mufson et al., 2003). We examined expression level differences of individual mitochondrial oxidative phosphorylation subunits at the transcript and encoded protein levels $\sim 6 \mathrm{MO}$, a timepoint where BFCN degeneration is initiated in Ts65Dn mice. Bioinformatic inquiry of our recent single population RNA-seq analysis of MSN BFCNs in Ts65Dn and 2N littermates indicates that oxidative phosphorylation and mitochondrial dysfunction are two of the top canonical pathways by Ingenuity Pathway Analysis (IPA) downregulated in this vulnerable cell type directly relevant to human DS and AD pathophysiology (Alldred et al., 2021). Understanding the age of degeneration 

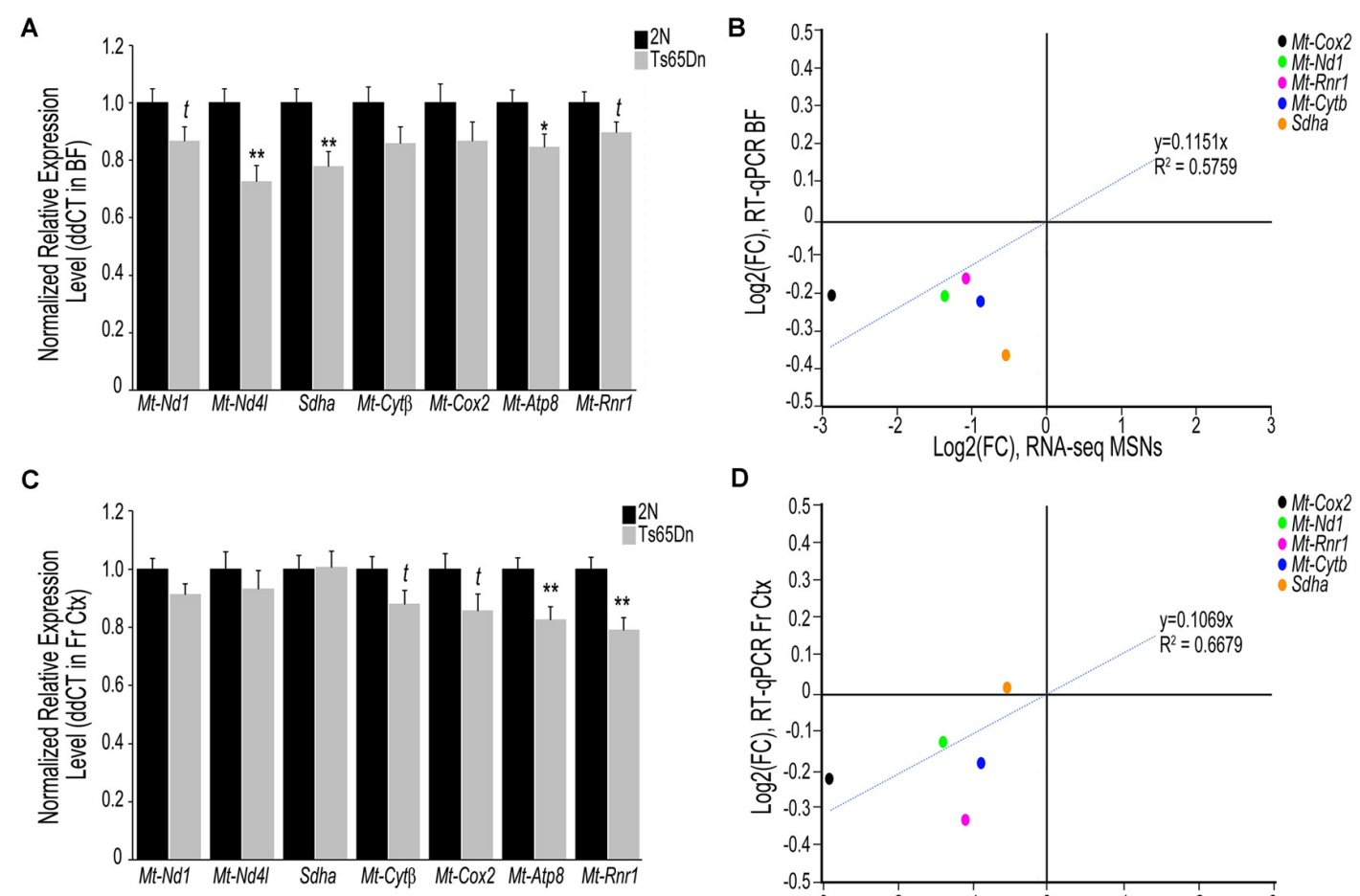

D

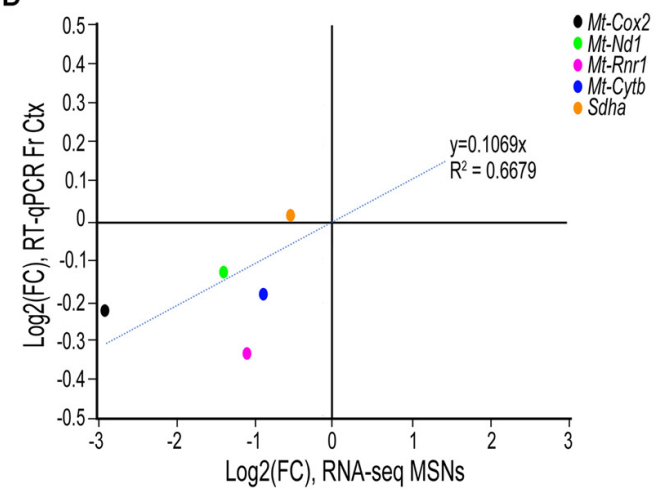

FIGURE 2 | Interrogation of oxidative phosphorylation Complex I-V subunit gene expression within the trisomic basocortical circuit. RT-qPCR was performed to determine gene expression levels using regional dissections of the basal forebrain (BF; $\mathbf{A}, \mathbf{B})$ and Fr Ctx (C,D) from Ts65Dn and 2N littermates at $\sim 6 \mathrm{MO}$ for six genes. (A) Bar graph represents ddCT of each gene normalized to $2 \mathrm{~N}$ levels in the BF. Significant downregulation was found for Mt-Nd4I, Sdha, and Mt-Atp8 and trend-level downregulation was found for Mt-Nd1, Mt-Cyt $\beta$, and Mt-Rnr1. (B) Correlation plot association between MSN BFCN RNA-seq LFC (x-axis) and BF RT-qPCR LFC (y-axis). (C) Bar graph represents ddCT of each gene normalized to $2 \mathrm{~N}$ levels in the Fr Ctx. Significant downregulation was found for Mt-Atp8 and Mt-Rnr1 and trend-level downregulation was found for Mt-CytB and Mt-Cox2. (D) Correlation plot association between MSN BFCN RNA-seq LFC (x-axis) and Fr Ctx RT-qPCR LFC (y-axis). While most genes were not significantly downregulated by RT-qPCR, they trended in the same direction (downregulation). Sdha was the only gene that did not correlate with RNA-seq results. Black bars represent relative $2 \mathrm{~N}$ expression and gray bars indicate Ts65Dn expression normalized to $2 \mathrm{~N}$ for each gene (standard error of mean (SEM) is indicated by error bars). Key: ${ }^{*} p<0.05,{ }^{* *} p<0.01 ; t$, trend.

onset and the pathways involved at this inception point would help pinpoint novel targets for therapeutic development for slowing or stopping the degeneration associated with cognitive decline and loss of executive function. Indeed, despite hundreds of trials for $\mathrm{AD}$ and $\mathrm{DS}$ treatment, no new therapeutics have proven effective for $\mathrm{AD}$ or $\mathrm{DS}$, with researchers suggesting that the delayed onset of treatment is a driving factor of the failure of these clinical trials (Gauthier et al., 2016; Yiannopoulou et al., 2019).

Oxidative stress and mitochondrial dysfunction are thought to play a critical role in DS and AD pathology (Mattson et al., 2008; Lott, 2012; Helguera et al., 2013; Izzo et al., 2018). Interestingly, a recent review postulates DS is an oxidative phosphorylation disorder (Bayona-Bafaluy et al., 2021). To date, the majority of studies evaluating oxidative phosphorylation and mitochondrial dysfunction in DS have used in vitro model systems. Although helpful to ascertain mechanistic interactions, these in vitro studies benefit from parallel in vivo assessments using the animal model and postmortem human brain tissue in the context of DS and AD for greater applicability and disease relevance.
Based on the present results, we postulate downregulation of select members of the oxidative phosphorylation pathway in the BF precedes degenerative changes within Fr Ctx, in a connectivity based degeneration course of action. Supporting evidence comes from the observation that downregulation of oxidative phosphorylation pathway complex genes is less pervasive in Fr Ctx along with a generalized lack of encoded protein changes at $\sim 6 \mathrm{MO}$, whereas the $\mathrm{BF}$ has profound transcript and protein level changes (Figures 2A,C, 3B,E). These results correlate strongly with our previous RNA-seq analysis (Figures 2B, 3B) within MSN BFCNs, indicating mitochondrial oxidative phosphorylation complexes are highly vulnerable in DS. Complementary observations were found in the Fr Ctx by an independent group using the Ts65Dn model at different age timepoints including no changes in Complex II (SDHB) or Complex V (ATP5A) subunits and with deficits in Complex III (UQCRC2) and Complex IV (MT-COX2) not seen until $18 \mathrm{MO}$ (Lanzillotta et al., 2021). Our $\sim 6 \mathrm{MO}$ data is commensurate with their $9 \mathrm{MO}$ data showing a significant increase in Complex I (NDUFB8) levels, which is reversed at $18 \mathrm{MO}$, possibly indicating data this independent research group postulates is 


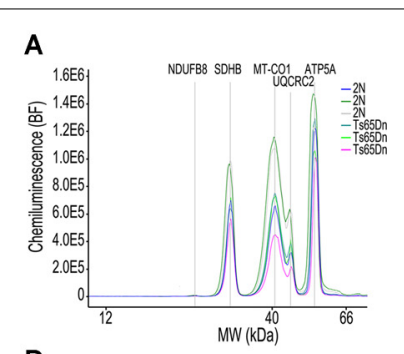

D

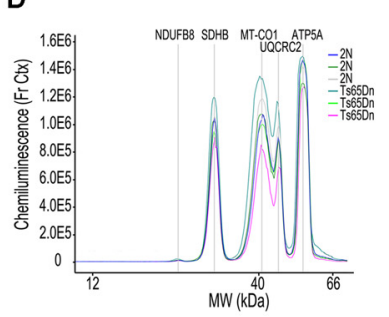

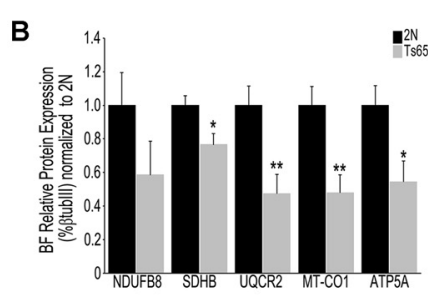

E

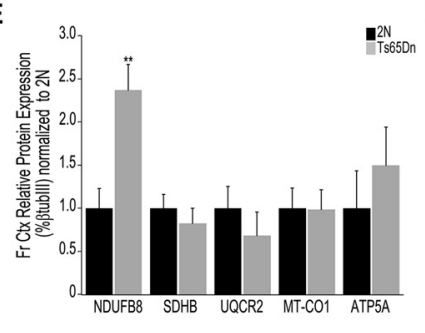

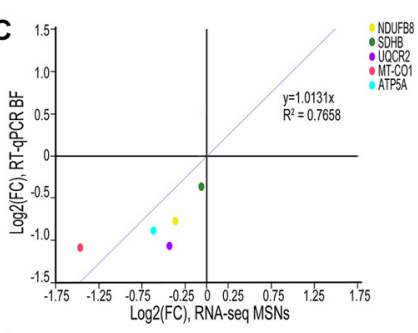

$F$

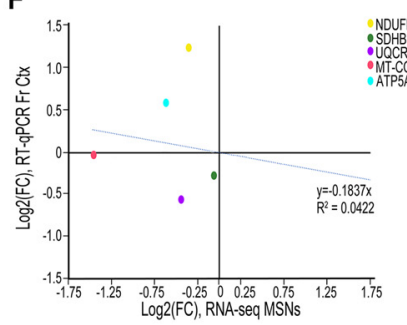

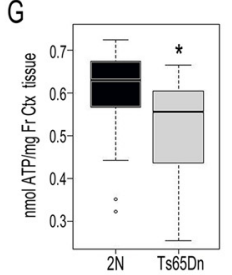

FIGURE 3 | Interrogation of oxidative phosphorylation Complex I-V subunit protein expression within the trisomic basocortical circuit. (A) Representative digital signatures of each assayed protein raw expression levels ( $\mathrm{y}$-axis) and molecular weight ( $\mathrm{x}$-axis) from Ts65Dn and 2N BF tissue homogenates. (B) Bar graph represents relative protein levels (normalized to beta-tubulin ( $\beta$ Tublli) as a percentage of $2 \mathrm{~N}$ expression in BF tissue homogenates. Black bars represent $2 \mathrm{~N}$ and gray bars indicate Ts65Dn (SEM indicated by error bars). (C) Correlation plot association between MSN BFCN RNA-seq LFC (x-axis) and BF protein level LFC (y-axis) indicating a high correlation. (D) Representative digital signatures of each assayed protein raw expression levels ( $\mathrm{y}$-axis) and molecular weight (x-axis) from Ts65Dn and $2 \mathrm{~N} \mathrm{Fr}$ Ctx tissue homogenates. (E) Bar graph represents relative protein levels (normalized to $\beta$ Tublll) as a percentage of $2 \mathrm{~N}$ expression in Fr Ctx tissue homogenates (SEM indicated by error bars). (F) Correlation plot association between MSN BFCN RNA-seq LFC (x-axis) and Fr Ctx protein level LFC (y-axis) indicating no significant correlation. (G) Box and whisker plots highlight downregulation of ATP levels in trisomic Fr Ctx. Key: ${ }^{\star} p<0.05$, ${ }^{\star \star} p<0.01$.

due to the shift from juvenile age to adulthood and to aged animals, whereby Ts65Dn mice react differently than their $2 \mathrm{~N}$ counterparts (Lanzillotta et al., 2021). These researchers also conclude the oxidative phosphorylation machinery is highly downregulated in their DS cohort (Lanzillotta et al., 2021). BF was not evaluated, so comparisons are only available in the Fr Ctx. Although a compensatory mechanism prior to overt pathology onset is possible and would explain upregulation of NDUFB8 protein levels in Fr Ctx, differential regulation of this subunit does not reflect overall deficits in the oxidative phosphorylation pathway seen in our $\sim 6 \mathrm{MO}$ trisomic cohort during initiation of BFCN degeneration. We also demonstrate downregulation of cholinergic and glutamatergic protein levels in the BF (Supplementary Figure 1), indicating synaptic deficits also exist in $\sim 6 \mathrm{MO}$ trisomic mice, correlating with single population RNA-seq findings (Alldred et al., 2021). Synapticrelated marker downregulation in the $\mathrm{BF}$ which project to the Fr Ctx, along with the high number of significantly downregulated genes and proteins evidenced in BF, and the reduction of corresponding deficits in Fr Ctx are supportive of our overarching hypothesis that BF degeneration drives oxidative phosphorylation dysregulation and precedes cortical dysfunction in DS as well as across the AD spectrum (Mufson et al., 2016, 2019). Deficits in the activity of the oxidative phosphorylation complex have been shown in astrocyte cultures derived from the Ts1Cje DS mouse model, including lower ATP levels and decreased mitochondrial membrane potential (Shukkur et al., 2006). This study also showed in vivo brain ATP levels are reduced in Ts1Cje mice at 3 MO (Shukkur et al., 2006). Neural progenitor cells obtained from the Ts65Dn hippocampus revealed reduced ATP levels and loss of mtDNA levels, indicating a neuronal deficit in energy production (Vacca et al., 2016; Valenti et al., 2018). We corroborate these findings within the Ts65Dn model in vivo, with reduction in ATP levels and overall mitochondrial RNA (via Mt-Rnr1) in Fr Ctx (Figures 2C, 3G). We postulate significant decreases in trisomic mitochondrial RNA, as determined by $M t-R n r 1$ may drive gene expression changes seen in Fr Ctx tissue. Interestingly, these deficits may be the result of the use of regional tissue with admixed cell types for the protein assays, with the expectation that neuron-specific protein level changes may be obscured. Conversely, BF oxidative phosphorylation changes are likely to involve multiple neuronal subtypes within the BF and are able to be discriminated even in admixed tissue.

Mitochondrial dysfunction beyond the oxidative phosphorylation pathway has been linked to DS pathology including deficits in mitochondrial biogenesis, turnover, and mitophagy (Helguera et al., 2013; Izzo et al., 2018; Bordi et al., 2019; Mollo et al., 2019, 2020). The balance between biogenesis and mitophagy is perturbed using in vitro models of DS, with hyperactivation of proteins from the rapamycin (mTOR) pathway responsible for mitophagy and impaired activity of PGC-1 $\alpha$ pathway responsible for biogenesis (Valenti et al., 2016; Bordi et al., 2019; Mollo et al., 2019, 2020). These studies link dysregulation of mitochondrial function to dysregulation of autophagy pathways, which have also been shown to be dysregulated in MSN BFCN neurons by single population RNA-seq (Alldred et al., 2021). We postulate BFCN degeneration in $\mathrm{DS}$ and $\mathrm{AD}$ may be directly related to the failure of mitochondrial turnover and mitophagy. From a translational 
perspective, choline, an essential nutrient required for the production of the cholinergic neurotransmitter acetylcholine, the phosphatidylethanolamine $\mathrm{N}$-methyltransferase (PEMT) pathway for generation of key substrates of neuronal membranes, and the primary methyl donor in the brain, requires properly functioning mitochondria and the oxidative phosphorylation pathway (Mailloux et al., 2016). Our collaborative group demonstrated metabolites of the PEMT pathway are significantly downregulated in the brains of Ts65Dn mice including within the BF and Fr Ctx (Yan et al., 2014), which also implicate deficits in mitochondria and the oxidative phosphorylation pathway. Further study on overall mitochondrial turnover, morphology, and mitophagy within in vivo DS and $\mathrm{AD}$ models are warranted.

RT-qPCR analysis and protein chemistry in trisomic BF and Fr Ctx reveals downregulation within oxidative phosphorylation genes and proteins that are specific to the brain regions analyzed. We recognize there are limitations and caveats to the studies we conducted using this approach. For example, each of these complexes in the oxidative phosphorylation pathway consists of numerous subunits, and the current interrogation was limited to one to two genes or proteins per complex. It is possible that other Complex I-V subunits will not be differentially regulated, although the larger number of changes identified by IPA and KEGG analysis from the MSN BFCN RNA-seq study of $\sim 6$ MO Ts65Dn mice and $2 \mathrm{~N}$ littermates analysis indicates $\mathrm{BFCNs}$ are significantly vulnerable and display an abundance of deficits in oxidative phosphorylation and mitochondrial function transcripts (Alldred et al., 2021). Another noted limitation is that when examining the correlation analysis between BF regional RT-qPCR and single population MSN BFCN RNA-seq, we were unable to compare $M t$ $N d 4 l$ or $M t-A t p 8$, as they were not detected by RNA-seq (Alldred et al., 2021), likely due to the small size of the fulllength mitochondrial RNA and low RNA input (McCormick et al., 2011; Stark et al., 2019). It is important to note this study was performed in male mice, and sex differences may exist in BFCN degenerative programs, as morphological differences between sexes in BFCNs have been demonstrated in trisomic mice (Kelley et al., 2014b). A cohort of female trisomic mice is currently being accrued for RNA-seq, RTqPCR, and protein-based analyses, enabling sex differences to be evaluated in future studies. Importantly, Lanzillotta et al. (2021) demonstrate expression level changes in the oxidative phosphorylation pathway in Fr Ctx within trisomic mice during aging. Although beyond the scope of the present study, an aging time-course assessment of the Ts65Dn BF is warranted in future studies. Moreover, future assessments are planned to evaluate vulnerable cell types, brain regions, age, and sex in

\section{REFERENCES}

ABI (2004). "Guide to performing relative quantitation of gene expression using real-time quantitative PCR," in Applied Biosystems Product Guide, 1-60.

Ahmed, M. M., Block, A., Tong, S., Davisson, M. M., and Gardiner, K. J. (2017). Age exacerbates abnormal protein expression in a mouse model of down trisomic models in relation to parallel observations found in human postmortem DS and AD studies in the same cell types and regions.

In conclusion, select dysregulation of oxidative phosphorylation pathway members is found at the RNA and encoded protein levels within the vulnerable basocortical circuit in an established model of DS and $\mathrm{AD}$ at a timepoint where BFCN degeneration is occurring. Defects appear to initiate in the BF and travel in the synaptic pathway that connects this vulnerable region to a cortical terminal field associated with memory and executive function. These data suggest deficits in oxidative phosphorylation in the $\mathrm{DS}$ and possibly $\mathrm{AD}$ brain may be circuit driven, and more specific to vulnerable brain regions than previously appreciated, especially in the context of neuropathological disorders and age-related cognitive decline.

\section{DATA AVAILABILITY STATEMENT}

The raw data supporting the conclusions of this article will be made available by the authors, without undue reservation.

\section{ETHICS STATEMENT}

The animal study was reviewed and approved by IACUC Nathan Kline Institute.

\section{AUTHOR CONTRIBUTIONS}

MJA GES, and SDG designed the experiments. MJA performed experiments. SHL, MJA, and SDG performed analysis of data. MJA and SDG wrote the manuscript. All authors contributed to the article and approved the submitted version.

\section{FUNDING}

Funding was provided by support from grants AG014449, AG043375, AG052524, and AG017617 from the National Institutes of Health.

\section{ACKNOWLEDGMENTS}

We thank Arthur Saltzman, M.S. for expert technical assistance.

\section{SUPPLEMENTARY MATERIAL}

The Supplementary Material for this article can be found online at: https://www.frontiersin.org/articles/10.3389/fnagi.2021.7079 50/full\#supplementary-material.

syndrome. Neurobiol. Aging 57, 120-132. doi: 10.1016/j.neurobiolaging.2017. 05.002

Alldred, M. J., Chao, H. M., Lee, S. H., Beilin, J., Powers, B. E., Petkova, E., et al. (2018). CA1 pyramidal neuron gene expression mosaics in the Ts65Dn murine model of down syndrome and Alzheimer's disease following maternal choline supplementation. Hippocampus 28, 251-268. doi: 10.1002/hipo.22832 
Alldred, M. J., Chao, H. M., Lee, S. H., Beilin, J., Powers, B. E., Petkova, E., et al. (2019). Long-term effects of maternal choline supplementation on CA1 pyramidal neuron gene expression in the Ts65Dn mouse model of down syndrome and Alzheimer's disease. FASEB J. 33, 9871-9884. doi: 10.1096/fj. 201802669RR

Alldred, M. J., Che, S., and Ginsberg, S. D. (2008). Terminal continuation (TC) RNA amplification enables expression profiling using minute RNA input obtained from mouse brain. Int. J. Mol. Sci. 9, 2091-2104. doi: 10.3390/ijms9112091

Alldred, M. J., Duff, K. E., and Ginsberg, S. D. (2012). Microarray analysis of CA1 pyramidal neurons in a mouse model of tauopathy reveals progressive synaptic dysfunction. Neurobiol. Dis. 45, 751-762. doi: 10.1016/j.nbd.2011.10. 022

Alldred, M. J., Lee, S. H., Petkova, E., and Ginsberg, S. D. (2015a). Expression profile analysis of vulnerable CA1 pyramidal neurons in young-middle-aged Ts65Dn mice. J. Comp. Neurol. 523, 61-74. doi: 10.1002/cne.23663

Alldred, M. J., Lee, S. H., Petkova, E., and Ginsberg, S. D. (2015b). Expression profile analysis of hippocampal CA1 pyramidal neurons in aged Ts65Dn mice, a model of down syndrome (DS) and Alzheimer's disease (AD). Brain Struct. Funct. 220, 2983-2996. doi: 10.1007/s00429-014-0839-0

Alldred, M. J., Penikalapati, S. C., Lee, S. H., Heguy, A., Roussos, P., and Ginsberg, S. D. (2021). Profiling basal forebrain cholinergic neurons reveals a molecular basis for vulnerability within the ts $65 \mathrm{dn}$ model of down syndrome and Alzheimer's disease. Mol. Neurobiol. doi: 10.1007/s12035-021-02453-3. [Epub ahead of print].

Annus, T., Wilson, L. R., Hong, Y. T., Acosta-Cabronero, J., Fryer, T. D., CardenasBlanco, A., et al. (2016). The pattern of amyloid accumulation in the brains of adults with down syndrome. Alzheimers Dement. 12, 538-545. doi: 10.1016/j. jalz.2015.07.490

Bambrick, L. L., and Fiskum, G. (2008). Mitochondrial dysfunction in mouse trisomy 16 brain. Brain Res. 1188, 9-16. doi: 10.1016/j.brainres.2007.10.045

Bayona-Bafaluy, M. P., Garrido-Perez, N., Meade, P., Iglesias, E., JimenezSalvador, I., Montoya, J., et al. (2021). Down syndrome is an oxidative phosphorylation disorder. Redox Biol. 41:101871. doi: 10.1016/j.redox.2021. 101871

Beacher, F., Daly, E., Simmons, A., Prasher, V., Morris, R., Robinson, C., et al. (2009). Alzheimer's disease and Down's syndrome: an in vivo MRI study. Psychol. Med. 39, 675-684. doi: 10.1017/S0033291708004054

Belichenko, P. V., Kleschevnikov, A. M., Masliah, E., Wu, C., TakimotoKimura, R., Salehi, A., et al. (2009). Excitatory-inhibitory relationship in the fascia dentata in the Ts65Dn mouse model of down syndrome. J. Comp. Neurol. 512, 453-466. doi: 10.1002/cne.21895

Belichenko, P. V., Masliah, E., Kleschevnikov, A. M., Villar, A. J., Epstein, C. J., Salehi, A., et al. (2004). Synaptic structural abnormalities in the Ts65Dn mouse model of down Syndrome. J. Comp. Neurol. 480, 281-298. doi: 10.1002/cne. 20337

Bittles, A. H., Bower, C., Hussain, R., and Glasson, E. J. (2007). The four ages of down syndrome. Eur. J. Public Health 17, 221-225. doi: 10.1093/eurpub/ckl103

Bordi, M., Berg, M. J., Mohan, P. S., Peterhoff, C. M., Alldred, M. J., Che, S., et al. (2016). Autophagy flux in CA1 neurons of Alzheimer hippocampus: increased induction overburdens failing lysosomes to propel neuritic dystrophy. Autophagy 12, 2467-2483. doi: 10.1080/15548627.2016. 1239003

Bordi, M., Darji, S., Sato, Y., Mellen, M., Berg, M. J., Kumar, A., et al. (2019). mTOR hyperactivation in down syndrome underlies deficits in autophagy induction, autophagosome formation and mitophagy. Cell Death Dis. 10:563. doi: 10.1038/s41419-019-1752-5

Briggs, C. A., Chakroborty, S., and Stutzmann, G. E. (2017). Emerging pathways driving early synaptic pathology in Alzheimer's disease. Biochem. Biophys. Res. Commun. 483, 988-997.doi: 10.1016/j.bbrc.2016.09.088

Cavedo, E., Lista, S., Houot, M., Vergallo, A., Grothe, M. J., Teipel, S., et al. (2020). Plasma tau correlates with basal forebrain atrophy rates in people at risk for Alzheimer disease. Neurology 94, e30-e41. doi: 10.1212/WNL. 0000000000008696

Cisterna, B., Sobolev, A. P., Costanzo, M., Malatesta, M., and Zancanaro, C. (2020). Combined microscopic and metabolomic approach to characterize the skeletal muscle fiber of the Ts65Dn mouse, a model of down syndrome. Microsc. Microanal. 26, 1014-1023. doi: 10.1017/S143192762002437X
Contestabile, A., Fila, T., Bartesaghi, R., and Ciani, E. (2006). Choline acetyltransferase activity at different ages in brain of Ts65Dn mice, an animal model for down's syndrome and related neurodegenerative diseases. J. Neurochem. 97, 515-526. doi: 10.1111/j.1471-4159.2006.03769.x

Cooper, J. D., Salehi, A., Delcroix, J. D., Howe, C. L., Belichenko, P. V., ChuaCouzens, J., et al. (2001). Failed retrograde transport of NGF in a mouse model of down's syndrome: reversal of cholinergic neurodegenerative phenotypes following NGF infusion. Proc. Natl. Acad. Sci. U S A 98, 10439-10444. doi: $10.1073 /$ pnas. 181219298

Costa, A. C. (2012). Alzheimer disease: treatment of Alzheimer disease in down syndrome. Nat. Rev. Neurol. 8, 182-184. doi: 10.1038/nrneurol.2012.40

Coyle, J. T., Oster-Granite, M. L., Reeves, R. H., and Gearhart, J. D. (1988). down syndrome, Alzheimer's disease and the trisomy16 mouse. Trends Neurosci. 11, 390-394. doi: 10.1016/0166-2236(88)90075-6

Coyle, J. T., Oster-Granite, M. L., and Gearhart, J. D. (1986). The neurobiologic consequences of down syndrome. Brain Res. Bull. 16, 773-787. doi: 10.1016/0361-9230(86)90074-2

Dick, M. B., Doran, E., Phelan, M., and Lott, I. T. (2016). Cognitive profiles on the severe impairment battery are similar in Alzheimer disease and Down syndrome with dementia. Alzheimer Dis. Assoc. Disord. 30, 251-257. doi: 10.1097/WAD.0000000000000132

Duchon, A., Raveau, M., Chevalier, C., Nalesso, V., Sharp, A. J., and Herault, Y. (2011). Identification of the translocation breakpoints in the Ts65Dn and Ts1Cje mouse lines: relevance for modeling down syndrome. Mamm. Genome 22, 674-684. doi: 10.1007/s00335-011-9356-0

Fernández-Cabello, S., Kronbichler, M., Van Dijk, K. R. A., Goodman, J. A., Spreng, R. N., and Schmitz, T. W. (2020). Basal forebrain volume reliably predicts the cortical spread of Alzheimer's degeneration. Brain 143, 993-1009. doi: 10.1093/brain/awaa012

Gauthier, S., Albert, M., Fox, N., Goedert, M., Kivipelto, M., Mestre-Ferrandiz, J., et al. (2016). Why has therapy development for dementia failed in the last two decades?. Alzheimers Dement. 12, 60-64. doi: 10.1016/j.jalz.2015.12.003

Ginsberg, S. D., Alldred, M. J., Counts, S. E., Cataldo, A. M., Neve, R. L., Jiang, Y., et al. (2010). Microarray analysis of hippocampal CA1 neurons implicates early endosomal dysfunction during Alzheimer's disease progression. Biol. Psychiatry. 68, 885-893. doi: 10.1016/j.biopsych.2010.05.030

Granholm, A. C., Sanders, L. A., and Crnic, L. S. (2000). Loss of cholinergic phenotype in basal forebrain coincides with cognitive decline in a mouse model of down's syndrome. Exp. Neurol. 161, 647-663. doi: 10.1006/exnr. 1999.7289

Grothe, M., Heinsen, H., and Teipel, S. (2013). Longitudinal measures of cholinergic forebrain atrophy in the transition from healthy aging to Alzheimer's disease. Neurobiol. Aging 34, 1210-1220. doi: 10.1016/j. neurobiolaging.2012.10.018

Hall, C. N., Klein-Flügge, M. C., Howarth, C., and Attwell, D. (2012). Oxidative phosphorylation, not glycolysis, powers presynaptic and postsynaptic mechanisms underlying brain information processing. J. Neurosci. 32, 8940-8951. doi: 10.1523/JNEUROSCI.0026-12.2012

Hartley, D., Blumenthal, T., Carrillo, M., DiPaolo, G., Esralew, L., Gardiner, K., et al. (2015). down syndrome and Alzheimer's disease: common pathways, common goals. Alzheimers Dement. 11, 700-709. doi: 10.1016/j.jalz.2014.10. 007

Helguera, P., Seiglie, J., Rodriguez, J., Hanna, M., Helguera, G., and Busciglio, J. (2013). Adaptive downregulation of mitochondrial function in down syndrome. Cell Metab. 17, 132-140. doi: 10.1016/j.cmet.2012.12.005

Hill, D. A., Gridley, G., Cnattingius, S., Mellemkjaer, L., Linet, M., Adami, H. O., et al. (2003). Mortality and cancer incidence among individuals with down syndrome. Arch. Intern. Med. 163, 705-711. doi: 10.1001/archinte.163.6.705

Holtzman, D. M., Santucci, D., Kilbridge, J., Chua-Couzens, J., Fontana, D. J., Daniels, S. E., et al. (1996). Developmental abnormalities and age-related neurodegeneration in a mouse model of down syndrome. Proc. Natl. Acad. Sci. US A 93, 13333-13338. doi: 10.1073/pnas.93.23.13333

Hunter, C. L., Bimonte, H. A., and Granholm, A. C. E. (2003a). Behavioral comparison of 4 and 6 month-old Ts65Dn mice: Age-related impairments in working and reference memory. Behav. Brain Res. 138, 121-131. doi: 10.1016/s0166-4328(02)00275-9

Hunter, C. L., Isacson, O., Nelson, M., Bimonte-Nelson, H., Seo, H., Lin, L., et al. (2003b). Regional alterations in amyloid precursor protein and nerve growth 
factor across age in a mouse model of down's syndrome. Neurosci. Res. 45, 437-445. doi: 10.1016/s0168-0102(03)00005-1

Iulita, M. F., Do Carmo, S., Ower, A. K., Fortress, A. M., Aguilar, L. F., Hanna, M., et al. (2014). Nerve growth factor metabolic dysfunction in Down's syndrome brains. Brain 137, 860-872. doi: 10.1093/brain/awt372

Izzo, A., Mollo, N., Nitti, M., Paladino, S., Cali, G., Genesio, R., et al. (2018). Mitochondrial dysfunction in down syndrome: molecular mechanisms and therapeutic targets. Mol. Med. 24:2. doi: 10.1186/s10020-018-0004-y

Jiang, Y., Mullaney, K. A., Peterhoff, C. M., Che, S., Schmidt, S. D., BoyerBoiteau, A., et al. (2010). Alzheimer's-related endosome dysfunction in down syndrome is Abeta-independent but requires APP and is reversed by BACE-1 inhibition. Proc. Natl. Acad. Sci. U S A 107, 1630-1635. doi: 10.1073/pnas. 0908953107

Kelley, C. M., Powers, B. E., Velazquez, R., Ash, J. A., Ginsberg, S. D., Strupp, B. J., et al. (2014a). Maternal choline supplementation differentially alters the basal forebrain cholinergic system of young-adult Ts65Dn and disomic mice. J. Comp. Neurol. 522, 1390-1410. doi: 10.1002/cne.23492

Kelley, C. M., Powers, B. E., Velazquez, R., Ash, J. A., Ginsberg, S. D., Strupp, B. J., et al. (2014b). Sex differences in the cholinergic basal forebrain in the Ts65Dn mouse model of down syndrome and Alzheimer's disease. Brain Pathol. 24, 33-44. doi: 10.1111/bpa.12073

Kim, S. H., Vlkolinsky, R., Cairns, N., Fountoulakis, M., and Lubec, G. (2001). The reduction of NADH ubiquinone oxidoreductase $24-$ and $75-\mathrm{kDa}$ subunits in brains of patients with down syndrome and Alzheimer's disease. Life Sci. 68, 2741-2750. doi: 10.1016/s0024-3205(01)01074-8

Kim, S. H., Vlkolinsky, R., Cairns, N., and Lubec, G. (2000). Decreased levels of complex III core protein 1 and complex $\mathrm{V}$ beta chain in brains from patients with Alzheimer's disease and down syndrome. Cell. Mol. Life Sci. 57, 1810-1816. doi: 10.1007/pl00000661

Lanzillotta, C., Tramutola, A., Giacomo, G. D., Marini, F., Butterfield, D. A., Domenico, F. D., et al. (2021). Insulin resistance, oxidative stress and mitochondrial defects in Ts65dn mice brain: A harmful synergistic path in down syndrome. Free Radic. Biol. Med. 165, 152-170. doi: 10.1016/j. freeradbiomed.2021.01.042

Lott, I. T. (2012). Neurological phenotypes for down syndrome across the life span. Prog. Brain Res. 197, 101-121. doi: 10.1016/B978-0-444-54299-1.00006-6

Lott, I. T., and Dierssen, M. (2010). Cognitive deficits and associated neurological complications in individuals with down's syndrome. Lancet Neurol. 9, 623-633. doi: 10.1016/S1474-4422(10)70112-5

Mai, C. T., Isenburg, J. L., Canfield, M. A., Meyer, R. E., Correa, A., Alverson, C. J., et al. (2019). National population-based estimates for major birth defects, 2010-2014. Birth Defects Res. 111, 1420-1435. doi: 10.1002/bdr2.1589

Mailloux, R. J., Young, A., Chalker, J., Gardiner, D., O’Brien, M., Slade, L., et al. (2016). Choline and dimethylglycine produce superoxide/hydrogen peroxide from the electron transport chain in liver mitochondria. FEBS Lett. 590, 4318-4328. doi: 10.1002/1873-3468.12461

Mann, D. M., Yates, P. O., and Marcyniuk, B. (1984). Alzheimer's presenile dementia, senile dementia of Alzheimer type and down's syndrome in middle age form an age related continuum of pathological changes. Neuropathol. Appl. Neurobiol. 10, 185-207. doi: 10.1111/j.1365-2990.1984.tb00351.x

Mattson, M. P., Gleichmann, M., and Cheng, A. (2008). Mitochondria in neuroplasticity and neurological disorders. Neuron 60, 748-766. doi: 10.1016/j. neuron.2008.10.010

McCormick, K. P., Willmann, M. R., and Meyers, B. C. (2011). Experimental design, preprocessing, normalization and differential expression analysis of small RNA sequencing experiments. Silence 2:2. doi: 10.1186/1758907X-2-2

McCulloch, C. E., Searle, S. R., and Neuhaus, J. M. (2011). Generalized, Linear and Mixed Models, 2nd Edn. New York, NY: John Wiley \& Sons.

Mesulam, M. M., Mufson, E. J., Levey, A. I., and Wainer, B. H. (1983). Cholinergic innervation of cortex by the basal forebrain: cytochemistry and cortical connections of the septal area, diagonal band nuclei, nucleus basalis (substantia innominata) and hypothalamus in the rhesus monkey. J. Comp. Neurol. 214, 170-197. doi: 10.1002/cne.902140206

Mollo, N., Cicatiello, R., Aurilia, M., Scognamiglio, R., Genesio, R., Charalambous, M., et al. (2020). Targeting mitochondrial network architecture in down syndrome and aging. Int. J. Mol. Sci. 21:3134. doi: $10.3390 /$ ijms 21093134
Mollo, N., Nitti, M., Zerillo, L., Faicchia, D., Micillo, T., Accarino, R., et al. (2019). Pioglitazone improves mitochondrial organization and bioenergetics in down syndrome cells. Front. Genet. 10:606. doi: 10.3389/fgene.2019.00606

Mufson, E. J., Counts, S. E., and Ginsberg, S. D. (2002). Gene expression profiles of cholinergic nucleus basalis neurons in Alzheimer's disease. Neurochem. Res. 27, 1035-1048. doi: 10.1023/a:1020952704398

Mufson, E. J., Counts, S. E., Ginsberg, S. D., Mahady, L., Perez, S. E., Massa, S. M., et al. (2019). Nerve growth factor pathobiology during the progression of Alzheimer's disease. Front. Neurosci. 13:533. doi: 10.3389/fnins.2019.00533

Mufson, E. J., Counts, S. E., Perez, S. E., and Ginsberg, S. D. (2008). Cholinergic system during the progression of Alzheimer's disease: therapeutic implications. Expert Rev. Neurother. 8, 1703-1718. doi: 10.1586/14737175.8.11.1703

Mufson, E. J., Ginsberg, S. D., Ikonomovic, M. D., and DeKosky, S. T. (2003). Human cholinergic basal forebrain: chemoanatomy and neurologic dysfunction. J. Chem. Neuroanat. 26, 233-242. doi: 10.1016/s08910618(03)00068-1

Mufson, E. J., Ikonomovic, M. D., Counts, S. E., Perez, S. E., Malek-Ahmadi, M., Scheff, S. W., et al. (2016). Molecular and cellular pathophysiology of preclinical Alzheimer's disease. Behav. Brain Res. 311, 54-69. doi: 10.1016/j.bbr.2016. 05.030

Nguyen, U., Squaglia, N., Boge, A., and Fung, P. A. (2011). The simple Western": a gel-free, blot-free, hands-free Western blotting reinvention. Nature Methods 8. doi: 10.1038/NMETH.F.353

Paxinos, G., and Franklin, K. B. J. (2001). The Mouse Brain in Stereotaxic Coordinates. Second Edition. San Diego: Academic Press.

Powers, B. E., Velazquez, R., Kelley, C. M., Ash, J. A., Strawderman, M. S., Alldred, M. J., et al. (2016). Attentional function and basal forebrain cholinergic neuron morphology during aging in the Ts65Dn mouse model of down syndrome. Brain Struct. Funct. 221, 4337-4352. doi: 10.1007/s00429-015$1164-\mathrm{y}$

Pratt, J. W. (1959). Remarks on zeros and ties in the wilcoxon signed rank procedures. J. Stat. Am. Assoc. 54, $655-667$.

Presson, A. P., Partyka, G., Jensen, K. M., Devine, O. J., Rasmussen, S. A., McCabe, L. L., et al. (2013). Current estimate of Down Syndrome population prevalence in the United States. J. Pediatr. 163, 1163-1168. doi: 10.1016/j.jpeds. 2013.06.013

Rueda, N., Florez, J., and Martinez-Cue, C. (2012). Mouse models of down syndrome as a tool to unravel the causes of mental disabilities. Neural Plast. 2012:584071. doi: 10.1155/2012/584071

Ruparelia, A., Pearn, M. L., and Mobley, W. C. (2012). Cognitive and pharmacological insights from the Ts65Dn mouse model of down syndrome. Curr. Opin. Neurobiol. 22, 880-886. doi: 10.1016/j.conb.2012. 05.002

Sendera, T. J., Ma, S. Y., Jaffar, S., Kozlowski, P. B., Kordower, J. H., Mawal, Y., et al. (2000). Reduction in TrkA-immunoreactive neurons is not associated with an overexpression of galaninergic fibers within the nucleus basalis in down's syndrome. J. Neurochem. 74, 1185-1196. doi: 10.1046/j.1471-4159. 2000.741185.x

Shukkur, E. A., Shimohata, A., Akagi, T., Yu, W., Yamaguchi, M., Murayama, M., et al. (2006). Mitochondrial dysfunction and tau hyperphosphorylation in Ts1Cje, a mouse model for down syndrome. Hum. Mol. Genet. 15, 2752-2762. doi: $10.1093 / \mathrm{hmg} / \mathrm{ddl} 211$

So, S. A., Urbano, R. C., and Hodapp, R. M. (2007). Hospitalizations of infants and young children with down syndrome: evidence from inpatient personrecords from a statewide administrative database. J. Intellect. Disabil. Res. 51, 1030-1038. doi: 10.1111/j.1365-2788.2007.01013.x

Stark, R., Grzelak, M., and Hadfield, J. (2019). RNA sequencing: the teenage years. Nat. Rev. Genet. 20, 631-656. doi: 10.1038/s41576-0190150-2

Strupp, B. J., Powers, B. E., Velazquez, R., Ash, J. A., Kelley, C. M., Alldred, M. J., et al. (2016). Maternal choline supplementation: a potential prenatal treatment for down syndrome and Alzheimer's disease. Curr. Alzheimer Res. 13, 97-106. doi: 10.2174/1567205012666150921100311

Teipel, S. J., Fritz, H. C., Grothe, M. J., and Alzheimer's Disease Neuroimaging, I. (2020). Neuropathologic features associated with basal forebrain atrophy in Alzheimer disease. Neurology 95, e1301-e1311. doi: 10.1212/WNL. 0000000000010192 
Vacca, R. A., Valenti, D., Caccamese, S., Daglia, M., Braidy, N., and Nabavi, S. M. (2016). Plant polyphenols as natural drugs for the management of down syndrome and related disorders. Neurosci. Biobehav. Rev. 71, 865-877. doi: 10.1016/j.neubiorev.2016.10.023

Valenti, D., de Bari, L., De Filippis, B., Henrion-Caude, A., and Vacca, R. A. (2014). Mitochondrial dysfunction as a central actor in intellectual disability-related diseases: an overview of down syndrome, autism, Fragile X and Rett syndrome. Neurosci. Biobehav. Rev. 46, 202-217. doi: 10.1016/j.neubiorev.2014.01.012

Valenti, D., de Bari, L., de Rasmo, D., Signorile, A., Henrion-Caude, A., Contestabile, A., et al. (2016). The polyphenols resveratrol and epigallocatechin-3-gallate restore the severe impairment of mitochondria in hippocampal progenitor cells from a down syndrome mouse model. Biochim. Biophys. Acta 1862, 1093-1104. doi: 10.1016/j.bbadis.2016. 03.003

Valenti, D., Braidy, N., De Rasmo, D., Signorile, A., Rossi, L., Atanasov, A. G., et al. (2018). Mitochondria as pharmacological targets in down syndrome. Free Radic. Biol. Med. 114, 69-83. doi: 10.1016/j.freeradbiomed.2017. 08.014

Wilcoxon, F. (1946). Individual comparisons of grouped data by ranking methods. J. Econ. Entomol. 39:269.doi: 10.1093/jee/39.2.269

Yan, J., Ginsberg, S. D., Powers, B., Alldred, M. J., Saltzman, A., Strupp, B. J., et al. (2014). Maternal choline supplementation programs greater activity of the phosphatidylethanolamine N-methyltransferase (PEMT) pathway in adult Ts65Dn trisomic mice. FASEB J. 28, 4312-4323. doi: 10.1096/fj.14251736
Yates, C. M., Simpson, J., Maloney, A. F., Gordon, A., and Reid, A. H. (1980). Alzheimer-like cholinergic deficiency in down syndrome. Lancet 2:979. doi: 10.1016/s0140-6736(80)92137-6

Yiannopoulou, K. G., Anastasiou, A. I., Zachariou, V., and Pelidou, S. H. (2019). Reasons for failed trials of disease-modifying treatments for Alzheimer disease and their contribution in recent research. Biomedicines 7:97. doi: 10.3390/biomedicines7040097

Conflict of Interest: The authors declare that the research was conducted in the absence of any commercial or financial relationships that could be construed as a potential conflict of interest.

Publisher's Note: All claims expressed in this article are solely those of the authors and do not necessarily represent those of their affiliated organizations, or those of the publisher, the editors and the reviewers. Any product that may be evaluated in this article, or claim that may be made by its manufacturer, is not guaranteed or endorsed by the publisher.

Copyright (c) 2021 Alldred, Lee, Stutzmann and Ginsberg. This is an open-access article distributed under the terms of the Creative Commons Attribution License (CC BY). The use, distribution or reproduction in other forums is permitted, provided the original author(s) and the copyright owner(s) are credited and that the original publication in this journal is cited, in accordance with accepted academic practice. No use, distribution or reproduction is permitted which does not comply with these terms. 